ARTICLE

Received 12 Aug 2013 | Accepted 28 Mar 2014 | Published 30 Apr 2014 DOl: 10.1038/ncomms4744

\title{
Robust and versatile ionic liquid microarrays achieved by microcontact printing
}

\author{
Christian A. Gunawan¹, Mengchen $\mathrm{Ge}^{1} \&$ Chuan Zhao ${ }^{1}$
}

Lab-on-a-chip and miniaturized systems have gained significant popularity motivated by marked differences in material performance at the micro-to-nano-scale realm. However, to fully exploit micro-to-nano-scale chemistry, solvent volatility and lack of reproducibility need to be overcome. Here, we combine the non-volatile and versatile nature of ionic liquids with microcontact printing in an attempt to establish a facile protocol for high throughput fabrication of open microreactors and microfluidics. The micropatterned ionic liquid droplets have been demonstrated as electrochemical cells and reactors for microfabrication of metals and charge transfer complexes, substrates for immobilization of proteins and as membrane-free high-performance amperometric gas sensor arrays. The results suggest that miniaturized ionic liquid systems can be used to solve the problems of solvent volatility and slow mass transport in viscous ionic liquids in lab-on-a-chip devices, thus providing a versatile platform for a diverse number of applications.

\footnotetext{
${ }^{1}$ School of chemistry, The University of New South Wales, Sydney, New South Wales 2052, Australia. Correspondence and requests for materials should be addressed to C.Z. (email: chuan.zhao@unsw.edu.au).
} 
ncreasing attention has been given to device-miniaturization technology over the past decades owing to its significant advantages for macro/lab-scale chemistry, such as material conservation, fast response or reaction time and high product yield $^{1-3}$. In addition, some violent reactions that are not feasible to be carried out on macroscale can be performed using lab-on-a-chip ${ }^{1}$. Nevertheless, device-miniaturization technologies face several challenges, of which solvent evaporation is one of the major issues ${ }^{2}$. Fast evaporation of volatile organic solvents in open microsystems is responsible for variation in solvent volume and change in concentration of components with time, resulting in unreliable products or analytical results. Furthermore, traditional organic solvents are prone to vapour cross-contamination, which might even cause the device to stop functioning properly ${ }^{3}$. Several approaches have been proposed to circumvent this issue, such as containing the solvent within channels or 'walls', which, however, are difficult to miniaturize and can suffer from problems such as large dead volume, obstruction of channels and poor control of hydrodynamic pressure ${ }^{2,4}$. Another suggested solution is to create an on-chip solvent reservoir in order to keep up with the evaporative losses ${ }^{5}$. This technique is also haunted with problems such as the large amount of space required for solvent storage, and is impractical for devices with multiple solvents ${ }^{3}$. The absence of standard apparatus forces most labs to develop their own custom components, which in turn makes such a device a one-of-a-kind with very little diversity or reproducibility ${ }^{5}$.

An alternative approach adopted here to address the issue is to use solvents that are non-volatile allowing fabrication of open micropatterns and microreactor systems, also called a 'wall-less' design. Ionic liquids (ILs) are a class of solvents with good thermal and chemical stability that typically have negligible vapour pressure $^{6}$. They have demonstrated applications in almost every field of chemistry, ranging from electrochemistry, organic synthesis to biological applications ${ }^{6}$. The values of non-volatile ILs in microsystems have been appreciated in several early studies. For example, Hozumi et al. ${ }^{7}$ have utilized ILs in photolithography as a medium for smart visualization of submicron-scale structures. Huang et al. ${ }^{8}$ applied ILs as electrolytes and developed a membrane-free amperometric gas sensor which can operate in extreme conditions such as high temperature and pressure. Dubois et al. ${ }^{2}$ have fabricated IL droplets $(300 \mathrm{nl})$ on a chip surface using Eppendorf micropipettes, and they demonstrated that efficient organic synthesis can be achieved inside the stable IL-droplet reactors 9 . Very recently, Jeong et al. ${ }^{10}$ applied a small drop of IL $(100 \mathrm{nl})$ as electrolyte to convert a metal oxide $\left(\mathrm{VO}_{2}\right)$ from an insulating to a conducting state, demonstrating the enormous potential of using non-volatile IL droplets for fabrication of non-volatile memory and logic chips.

Fostered by diverse applications of ILs and lab-on-a-chip devices, it is desirable to develop microfabrication protocols for IL-based miniature devices with high reproducibility and high throughput. Herein we report a facile and reproducible approach for fabrication of IL patterns using microcontact printing $(\mu \mathrm{CP})$, and report its use in the development of a gas sensor. $\mu \mathrm{CP}$ is a popular microfabrication method that can generate patterns with molecular-level detail, high fidelity, low cost and high scalability $^{11}$. However, $\mu$ CP methodology for ILs has not been established so far. In this study, we combine $\mu \mathrm{CP}$ with ILs and fabricate a series of IL micropatterns on gold and glass substrates. Several printing strategies have been established and tailored to ILs used in specific applications. We demonstrate that microfabrication of IL micropatterns gives versatile platforms for a range of applications.

\section{Results}

$\boldsymbol{\mu C P}$ of ILs. A standard $\mu \mathrm{CP}$ method is applied to transfer 11-mercaptoundecanoic acid (MUA) onto a gold-coated glass slide to form hydrophilic zones as a result of the terminal $-\mathrm{COOH}$ groups. Gaps are refilled with hexadecanethiol (HDT) to form hydrophobic, $\mathrm{CH}_{3}$-terminated zones (Supplementary Fig. 1) ${ }^{11}$. A drop of air-equilibrated IL (for example, 1-butyl-3methyl immidazolium tetrafluoroborate, $[\mathrm{BMIM}]\left[\mathrm{BF}_{4}\right]$ ) is brought into contact with the printed area for $1-2 \mathrm{~s}$ and excessive IL is removed by back suction. IL selectively attaches to the hydrophilic zones and forms arrays of IL droplets with uniform shape and size throughout the entire printed area that is shown in Fig. 1a-c. Detailed investigation on microdroplets shows that a very thin film of IL is formed on the microstructures with clear straight edges (Fig. 1b), attributed to the well-defined surface chemistry of the underlying self-assembled monolayers (SAMs). The film thickness in the middle of the droplet was measured as $9 \pm 1 \mu \mathrm{m}$ by scanning confocal microscopy. A range of ILs including aprotic ILs (for example, $[\mathrm{BMIM}]\left[\mathrm{BF}_{4}\right]$ and $[\mathrm{BMIM}]\left[\mathrm{PF}_{6}\right]$ ) and protic ILs (for example, ethylammonium nitrate, ethylammonium nitrate (EAN) and triethylammonium methylsulphonate (TAMS)) with distinctly different hydrophobicity, viscosity and acidity have been applied and each forms good quality patterns. It is noteworthy that, regardless of the hydrophobicity of the ILs (for example, $[\mathrm{BMIM}]\left[\mathrm{BF}_{4}\right]$ is hydrophilic and $[\mathrm{BMIM}]\left[\mathrm{PF}_{6}\right]$ is hydrophobic), they all adsorb preferentially to regions of MUA. This is attributed to different strengths of intermolecular forces between two surfaces, which generally follow a trend of hydrophilic/hydrophilic $>$ hydrophilic/ hydrophobic $>$ hydrophobic/hydrophobic ${ }^{12}$, and so even a hydrophobic IL will bond to the hydrophilic surface in preference to the hydrophobic areas. The approach can be easily adapted for fabrication of more complex two-dimensional microstructures (Supplementary Fig. 2a), which are useful for applications requiring microreactors and microfluidics.

Applying an IL onto a gold surface patterned with HDT SAMs, without filling the gap with MUA, also results in stable IL micropatterns (Fig. 1d). The IL droplet formed via this 'partial printing' strategy (Supplementary Fig. 3) is in direct contact with the bare gold surface and could be used as open microelectrochemical cells. It is also possible to form an IL array by directly using an IL as the ink. However, IL patterns formed via this approach are generally less uniform in size and shape (Supplementary Fig. 2b), and mechanically unstable owing to the weak interaction between an IL and the bare gold surface. To enhance the interaction between an IL and a gold substrate, we developed a 'semi-direct' approach by dissolving a thiol into the IL to be printed (Supplementary Fig. 4), and hypothesizing the formation of thiol-gold bonds, and then IL-thiol interactions will help to anchor the ILs onto the surface and enhance the stability of IL patterns. Previous studies also have used ILs as solvents for dissolution of thiols, and showed that structurally more stable SAMs can be formed on a gold surface ${ }^{13}$. Supplementary Figure $2 \mathrm{c}$ shows micropatterns of $[\mathrm{BMIM}]\left[\mathrm{PF}_{6}\right]$ containing dissolved MUA fabricated by the 'semi-direct' approach. The patterns formed by this approach are more uniform and physically more stable compared with those obtained through 'direct printing'.

The 'semi-direct' printing results give us the confidence that 'direct printing' of ILs is possible if strong IL-surface interactions or bonding can be established. To demonstrate that 'direct printing' of ILs is achievable, and also that ILs can be printed onto substrates other than gold, two protic ILs, ethylenediammonium acetate (EDAA) and ethylenediammonium formate (EDAF), were prepared according to the method described previously ${ }^{14}$ and applied as ink for direct printing onto glass (Supplementary Fig. 5). 

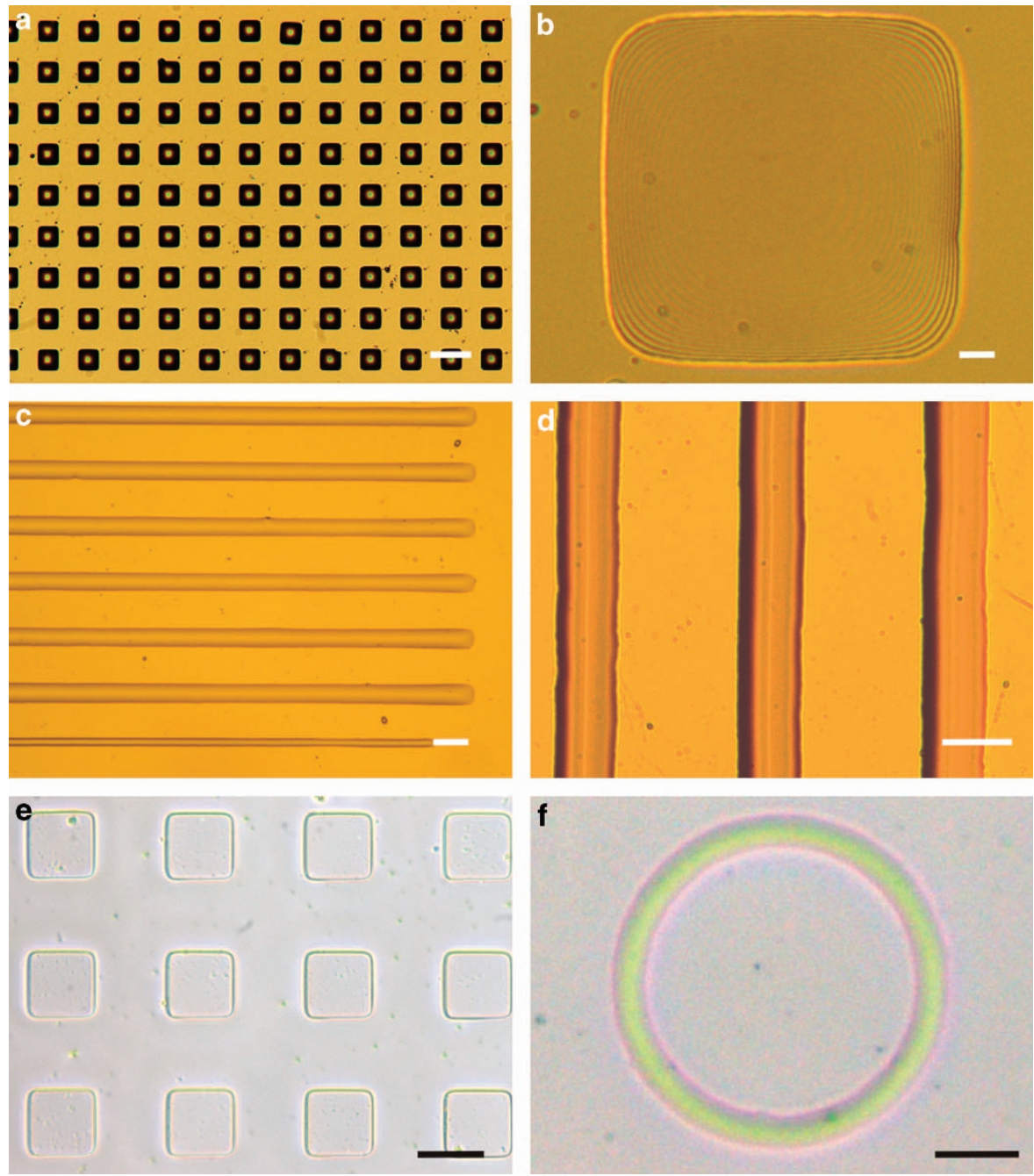

Figure 1 | Images of ionic liquid micropatterns. (a-d) Reflection micrographs of micropatterns of [BMIM][BF $\left.{ }_{4}\right]$ fabricated on a gold surface via (a-c) indirect printing (scale bar, $100 \mu \mathrm{m}(\mathbf{a}), 10 \mu \mathrm{m}$ (b) and $100 \mu \mathrm{m}(\mathbf{c})$ ) and (d) partial printing (scale bar, $30 \mu \mathrm{m}$ ); (e,f) Phase contrast micrographs of micropatterns of a protic IL [EDA][acetate] fabricated on a glass surface via direct printing (scale bar, $50 \mu \mathrm{m}(\mathbf{e})$ and $25 \mu \mathrm{m}(\mathbf{f})$ ).

The EDA cation in each IL contains two amine groups. A microscopic glass slide was pre-coated with -COOHterminated SAMs by treatment with phosphonohexadecanoic acid (PHDA) ${ }^{15}$. Figure 1e,f shows that well-defined, mechanically stable IL micropatterns are obtained on glass substrates by amine-carboxylic acid cross-linking ${ }^{16}$. It is noteworthy that ILs are 'designable' solvents and can be functionalized with specific groups for specific tasks such as thiol-functionalized ILs for attachment onto the gold surface ${ }^{17}$. We anticipate that direct printing can be particularly useful for micropatterning of taskspecific ILs by employing a range of surface cross-linking chemistry $^{16}$.

Characterization of IL micropatterns. We estimate the volume of IL in a single microdroplet. Typically, for a $50 \mu \mathrm{m}$ diameter circular droplet, assuming a spherical cap shape and a height of $10 \mu \mathrm{m}$ in the centre, the volume is calculated to be $9.2 \mathrm{pl}$, which corresponds to a surface-to-volume ratio of $2.4 \times 10^{3} \mathrm{~cm}^{2} \mathrm{l}^{-1}$. This volume is $>5$ orders of magnitude smaller than that can normally be prepared by using a micropipette ${ }^{2}$. The small volume and large surface-to-volume ratio are not only advantageous for conserving ILs, which are relatively costly materials, but also for a number of gas adsorption-based applications such as gas sensing or $\mathrm{CO}_{2}$ capture ${ }^{18,19}$. Further reduction in droplet volume can be achieved by decreasing the size of microstructures and/or using spin coating to achieve thinner IL droplets (Supplementary Fig. 2d). [BMIM] $\left[\mathrm{BF}_{4}\right]$ micropatterns obtained by indirect printing' show excellent mechanical stability in air (they survive when sample slides are tilted, turned upside down or even thrown from a height) and thermal stability up to $50{ }^{\circ} \mathrm{C}$. Intriguingly, hydrophilic $[\mathrm{BMIM}]\left[\mathrm{BF}_{4}\right]$ microdroplets are found to be stable when immersed into a range of nonpolar solvents. Figure 2 shows that $[\mathrm{BMIM}]\left[\mathrm{BF}_{4}\right]$ micropatterns remain unchanged when repetitively immersed in hexane. Infrared (IR) spectra recorded on the microdroplet before and after immersion into hexane are indistinguishable, showing characteristic bands of $[\mathrm{BMIM}]\left[\mathrm{BF}_{4}\right]$ at $2,878,2,940,2,966,3,122$ and $3,162 \mathrm{~cm}^{-1}$, respectively ${ }^{20}$. These liquid-stable IL microdroplets may find applications in understanding the fundamental properties of micro-scale interfaces between immiscible liquids, as well as a range of applications at liquid-liquid interfaces such as electrochemical sensors, solvent extraction, pharmacokinetics and solar energy conversion $^{21}$.

Metal electrodeposition from IL micropatterns. The micropatterned IL droplets provide a versatile platform for a range of applications. We demonstrate first the application of IL droplets as open electrochemical cells for microelectrodeposition of 

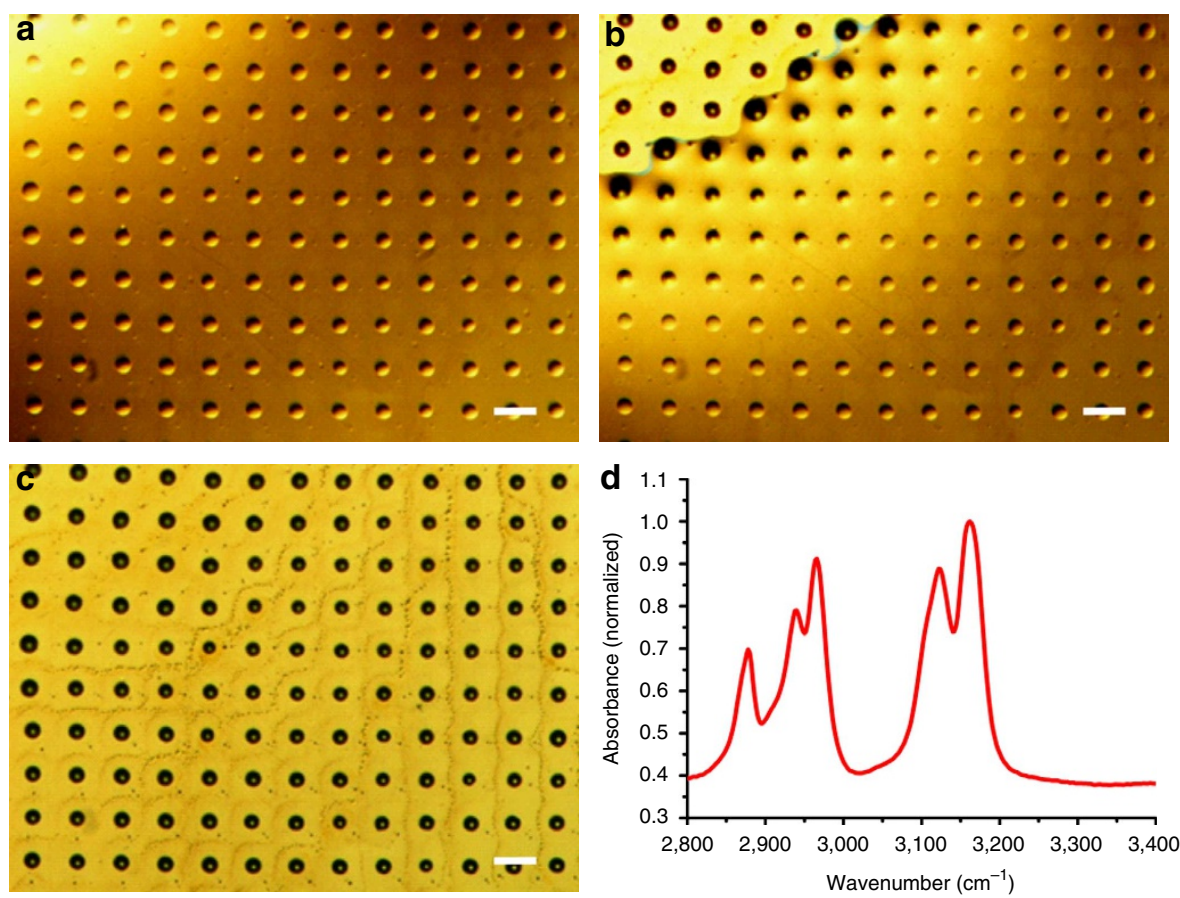

Figure 2 | Stable ionic liquid microarray immersed in a nonpolar solvent. (a-c) Reflection micrographs of $\left[\mathrm{BMIM}^{\mathrm{a}}\left[\mathrm{BF}_{4}\right]\right.$ on a gold surface when submerged in hexane (scale bar, $100 \mu \mathrm{m}$ ) (a), during evaporation (scale bar, $100 \mu \mathrm{m}$ ) (b) and after the complete evaporation of hexane (scale bar, $100 \mu \mathrm{m}$ ) (c); (d) Fourier transform infrared spectra recorded from a droplet in (c) confirming that the droplet is $[B M I M]\left[B F_{4}\right]$.

technologically important metals such as $\mathrm{Ag}$ and $\mathrm{Cu}$ (Supplementary Fig. 6) ${ }^{22-25}$. A protic IL (for example, EAN and TAMS) containing dissolved $\mathrm{Ag}^{+}$or $\mathrm{Cu}^{2}+$ is patterned on a gold substrate via the 'partial printing' method. Protic ILs are chosen because of the high solubilities of $\mathrm{Ag}^{+}$and $\mathrm{Cu}^{2+24,25}$. Cyclic voltammograms were recorded at a gold electrode to identify the electrodeposition potentials $\left(E_{\mathrm{dep}}\right)$ (Supplementary Fig. 7). Controlled potential deposition of Ag was then carried out at $E_{\text {dep }}=-0.1 \mathrm{~V}$ versus $\mathrm{Ag}$ quasi-reference electrode (QRE) for $60 \mathrm{~s}$, to form well-defined silver micropatterns, as confirmed with scanning electron microscopy (SEM) and energy dispersive X-ray (EDX) analysis (Fig. 3a; Supplementary Fig. 8). Electrodeposition of $\mathrm{Cu}$ performed at $E_{\mathrm{dep}}=-0.2 \mathrm{~V}$ versus $\mathrm{Cu} \mathrm{QRE}$ for $60 \mathrm{~s}$ also leads to formation of a $\mathrm{Cu}$ micropattern on gold (Supplementary Figs 9 and 10). These experiments prove the concept of using micropatterned IL droplets as templated electrochemical cells for fabrication of metal microstructures on a surface, which could potentially be used for fabrication of integrated circuits and interconnects.

Microfabrication of charge-transfer complexes AgTCNQ. We further demonstrate the utilization of the IL microdroplets for fabrication of charge-transfer complexes of silver tetracyanoquinodimethane (AgTCNQ), an attractive organic semiconductor for non-volatile memory because of its reproducible nanosecond electrical and optical switching behaviour and memory effects ${ }^{23,26}$. AgTCNQ can be fabricated via two routes from IL microdroplets. The first 'electrocrystallization' route employs open $[\mathrm{BMIM}]\left[\mathrm{BF}_{4}\right]$ micro-electrochemical cells containing equimolar $(3.33 \mathrm{mM}) \mathrm{Ag}^{+}$and $\mathrm{TCNQ}^{23}$. Controlled potential crystallization at $-0.8 \mathrm{~V}$ for $300 \mathrm{~s}$ forms AgTCNQ by the spontaneous reaction between dissolved TCNQ and the electrodeposited Ag metal (Supplementary Fig. 11). AgTCNQ micropatterns were examined by SEM (Fig. 3b). EDX analysis reveal electrocrystallized materials containing $\mathrm{Ag}, \mathrm{C}$ and $\mathrm{N}$, as expected for AgTCNQ formation ${ }^{23}$. In the second route, AgTCNQ is fabricated by photocrystallization ${ }^{26}$, using the IL microdroplets as photoreactors (Supplementary Fig. 12). In this approach, [BMIM] $\left[\mathrm{BF}_{4}\right]$ containing equimolar $(3.33 \mathrm{mM}) \mathrm{Ag}^{+}$ and TCNQ and 10\% volume fraction benzyl alcohol as a sacrificial electron donor was patterned onto a gold surface via 'indirect printing'. Irradiating the IL patterns at $\lambda=425 \mathrm{~nm}$ for $5 \mathrm{~min}$ led to the detection of visible colour change of the IL-patterned zone from yellow to blue, and then dark blue with extended irradiation times. This is consistent with previous observations $^{26}$, and is attributed to the photoreduction of TCNQ to $\mathrm{TCNQ}^{-}$radical anions, which react with $\mathrm{Ag}^{+}$forming dark blue AgTCNQ crystals, as confirmed by microscopy investigation (Supplementary Fig. 13). These data show the promise of using an array of IL microdroplets as photoreactors for screening a range of photochemical reactions in ILs ${ }^{26,27}$.

Protein micropatterning using ILs as immobilization media. ILs have demonstrated considerable applications for biocatalysis, biotransformations, protein-based pharmaceutical preparations and cellular therapies ${ }^{28}$. While many ILs are not biocompatible, some have been shown to be useful media for solubilization, immobilization and stabilization of biological molecules such as enzymes, DNA and proteins with retained activities ${ }^{29}$. These ILs can thus be used for micropatterning of biomolecules that have high stability by simply dissolving in the ILs and then printing as microdroplets onto a surface. Figure $3 c$ illustrates the fabrication of protein microarrays of cytochrome $c$ (Cyt $c$ ), a highly studied redox protein, which can be solubilized and remain active even in dry ILs ${ }^{30}$. A $10-\mu \mathrm{M}$ solution of Cyt $c$-OG488 (Oregon Green ${ }^{\circledR}$ 488) was prepared in $[\mathrm{BMIM}]\left[\mathrm{BF}_{4}\right]$ and then deposited on a gold chip patterned with $50 \mu \mathrm{m}$ circles by 'indirect printing'. Fluorescence images confirm the protein is patterned onto the surface and distributed evenly in the IL droplets. In another example, we patterned $[\mathrm{BMIM}]\left[\mathrm{BF}_{4}\right]$ containing dispersed bovine 

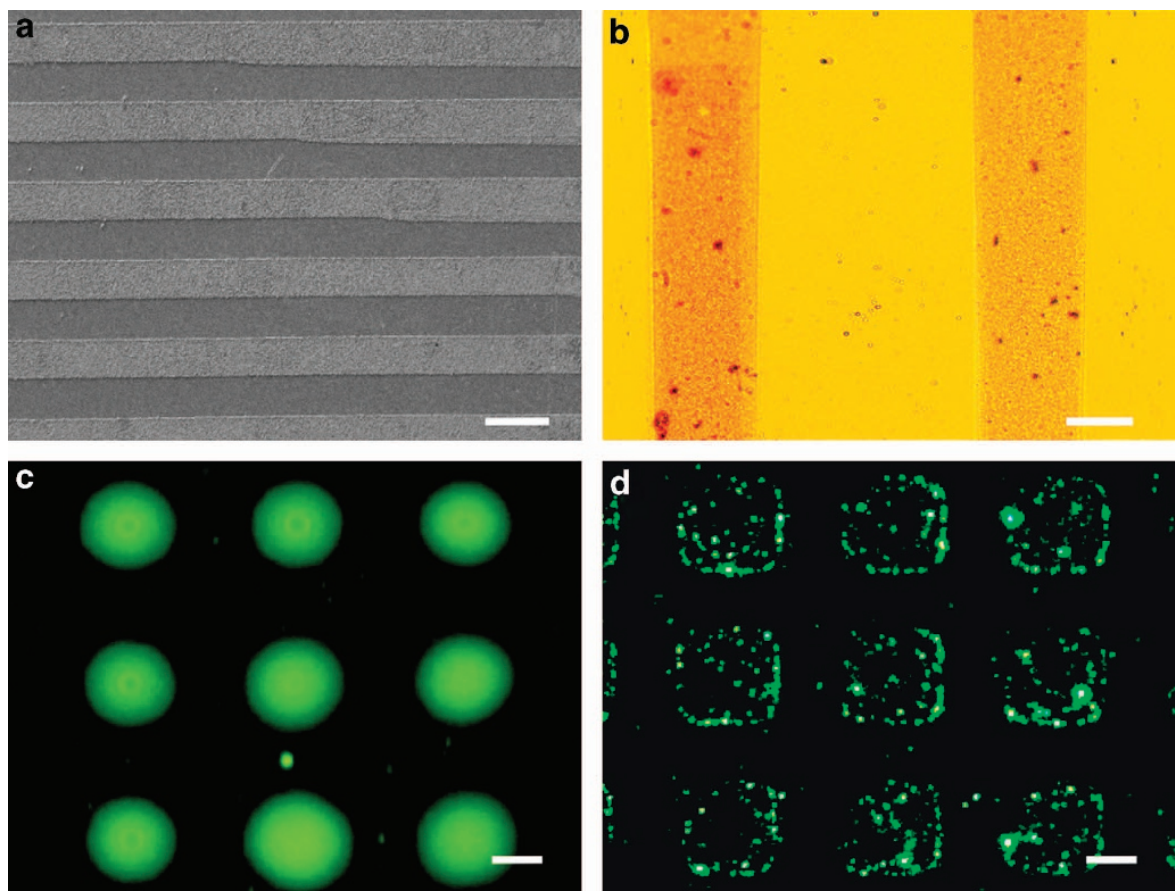

Figure 3 | Applications of ionic liquid micropatterns. (a) SEM micrograph of silver patterns electrodeposited onto a gold substrate from microprinted EAN containing $1 \mathrm{M} \mathrm{Ag}^{+}$(scale bar, $100 \mu \mathrm{m}$ ); (b) micrograph of AgTCNQ electrodeposited from micropatterned [BMIM][BF $\left.{ }_{4}\right]$ from $3.33 \mathrm{mM} \mathrm{Ag}{ }^{+}$ and $3.33 \mathrm{mM}$ TCNQ for $300 \mathrm{~s}$ (scale bar, $25 \mu \mathrm{m}$ ); (c,d) fluorescence micrographs of micropatterned [BMIM][BF 4 containing dissolved (c) $10 \mu \mathrm{M}$ Cyt c-OG488 (scale bar, $25 \mu \mathrm{m}$ ) and (d) $0.753 \mu \mathrm{M}$ BSA-FITC (scale bar, $25 \mu \mathrm{m}$ ), respectively.

serum albumin/fluorescein isothiocyanate conjugate (BSAFITC). The fluorescent images in Fig. $3 \mathrm{~d}$ also show the immobilization of BSA within the IL droplets. Further study is under way to investigate the activity of proteins inside the IL droplets and to explore the use of these IL-based protein arrays for analytical/screening purposes. In addition, the versatility of ILs is such that their properties can be tailored to different biological reactions by mixing ILs with water, other ILs or organic solvents $^{29,31}$ all of which can be readily patterned using $\mu \mathrm{CP}$ (Supplementary Fig. 14).

Development of an amperometric microarray gas sensor. Finally, we demonstrate a micropatterned IL interface as a unique platform for gas sensing. ILs have been regarded as ideal candidate electrolytes for replacing aqueous/organic solvent electrolytes in commercial gas sensors, which are prone to drying out and sensor failure, and for the development of membranefree gas sensors (Supplementary Fig. 15a,b) ${ }^{8,32}$. However, current IL-based gas sensors suffer from one intrinsic drawback, namely the high viscosity of ILs ${ }^{32}$. The diffusion coefficients of gases (for example, $\mathrm{O}_{2}$ ) in ILs are usually 1-2 orders of magnitude smaller than in aqueous or organic solvents ${ }^{33,34}$. This can significantly lower the sensitivity and response times of the sensors. Herein, we apply microcontact-printed IL droplets as a sensor for gaseous oxygen. Each IL microdroplet on the electrode serves as a sensing unit, forming a microarray gas sensor (Supplementary Fig. 15c,d). This sensor design benefits from the extremely small volume and thickness of each droplet, so diffusion distance is small and slow diffusion is not a significant issue. Furthermore, the extremely large surface-to-volume ratio, and high current density all help to improve the sensitivity, response time and overall quality of the signal from the sensors.

Figure 4a shows the amperometric current-time traces obtained at a $[\mathrm{BMIM}]\left[\mathrm{NTf}_{2}\right]$-based microarray sensor in comparison with a conventional macroelectrode-based IL gas sensor for oxygen sensing. Lower background current is first observed for the microarray sensors, which is somewhat expected, as its electrode surface area is only ca. $1 / 3$ of the macroelectrode. On the injection of $\mathrm{O}_{2}\left(5,400\right.$ p.p.m., $\left.\mu \mathrm{ll}^{-1}\right)$, the current increases as $\mathrm{O}_{2}$ is reduced at the applied potential $(-1.2 \mathrm{~V}$ versus $\mathrm{Ag})$. Remarkably, although the electrode surface area is smaller, the current obtained at the microarray sensor is significantly higher than the macroelectrode sensor. Taking into account the surface area, the current density obtained at the microarray sensor was found to be five times higher than the macro-IL sensor (Supplementary Fig. 17). The high sensitivity could be attributed to the extremely large surface-to-volume ratio (fast gas adsorption) and small diffusion distance in each droplet. For better comparison of response time, the current response is further normalized by the plateau current (I/Is) (Fig. 4b) and the response times for the microarray sensor (25.1 s and $45.2 \mathrm{~s}$ to $50 \%$ and $90 \%$ of the plateau, respectively) are about $10 \mathrm{~s}$ faster than the macroelectrode sensor. The microarray sensor shows well-defined responses to different concentrations of $\mathrm{O}_{2}$ and excellent reproducibility on exposure to constant $\mathrm{O}_{2}$ concentration (5,400 p.p.m.) with a relative sample s.d. of $1.0 \% \quad(n=7$, Fig. 4c). The calibration curve shown for the microarray sensor in Fig. 4d exhibits excellent linearity over a wide concentration range of $\mathrm{O}_{2}$, and is significantly more sensitive than the macroelectrode sensor (curve slopes of $1 \mathrm{nA} \mathrm{cm}{ }^{-2}$ per p.p.m. versus $0.07 \mathrm{nA} \mathrm{cm}^{-2}$ per p.p.m.) (Table 1). The superior performance (for example, high sensitivity, fast response time and high reproducibility) of the microarray sensor is attributed to the much smaller and thinner IL droplets that can be prepared using $\mu \mathrm{CP}$, which allows faster access of oxygen to the electrode surface despite the higher viscosity, and the non-volatile nature of ILs, as well as the mechanic stability of the IL micropatterns. It is worth noting that the membrane-free sensor design does not imply that the ILs will be exposed directly to an open atmosphere. 
a

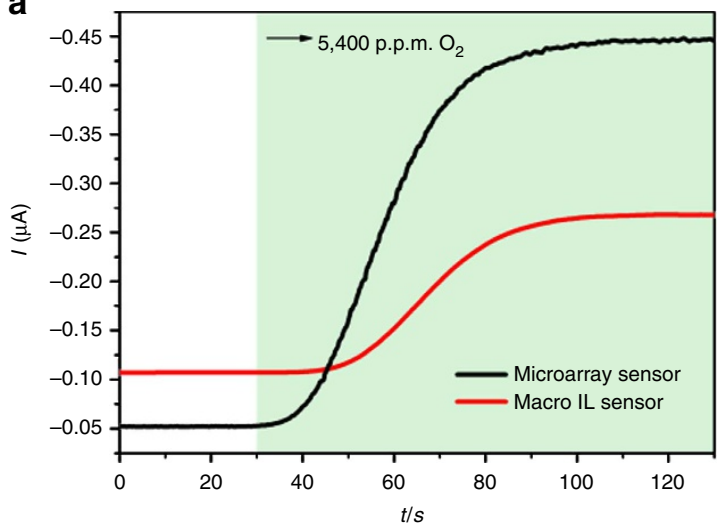

C

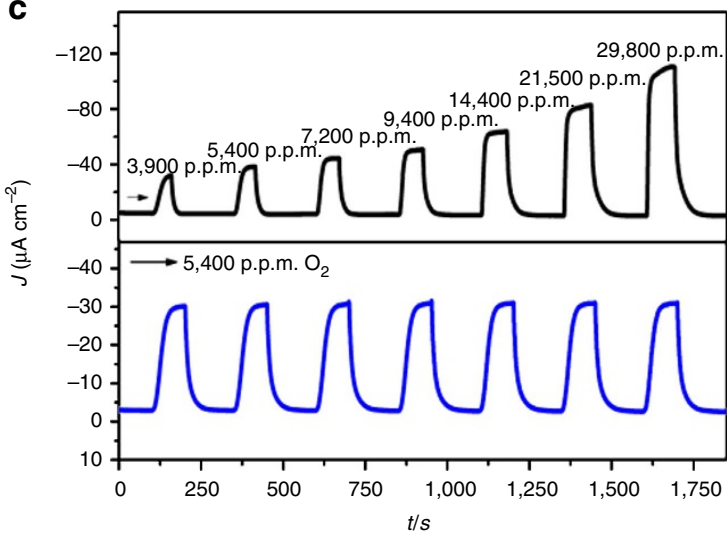

b

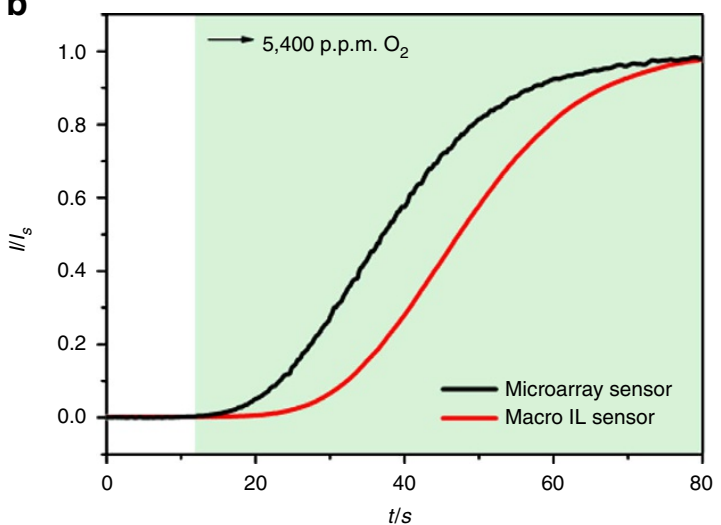

d

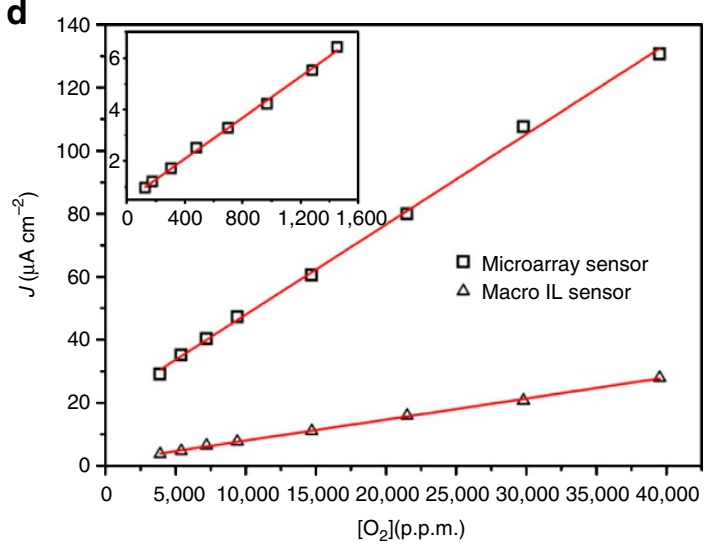

Figure 4 | Micropatterned IL droplets as amperometric gas sensor. (a) Amperometric current-time $(I-t)$ and $(\mathbf{b})$ normalized $\left(I / I_{s}\right)$ current-time responses of a IL microarray gas sensor versus a macrodisc electrode-based IL sensor, on exposure of 5,400 p.p.m. $\mathrm{O}_{2}$ as indicated by the green background; (c) Current density-time $(J-t)$ responses of a microarray sensor to different concentrations of $\mathrm{O}_{2}$ (top) and replicate exposures to 5,400 p.p.m. $\mathrm{O}_{2}$ (bottom); (d) calibration curves for a microarray sensor versus a macro-IL sensor. The inset is a calibration curve for a microarray sensor over a low concentration range (130-1,450 p.p.m.), where the macro-IL sensor shows no response.

\begin{tabular}{|c|c|c|c|c|c|}
\hline Sensor type & $\begin{array}{l}\text { Linear range } \\
\text { (p.p.m.) }\end{array}$ & Calibration equation & $R^{2}$ & $\begin{array}{c}\text { LOD }^{\star} \\
\text { (p.p.m.) }\end{array}$ & $\begin{array}{c}\mathrm{LOQ}^{\star} \\
\text { (p.p.m.) }\end{array}$ \\
\hline Microarray sensor & $130-1,450$ & $J\left(\mathrm{Acm}^{-2}\right)=-5.00 \times 10^{-7} \mathrm{Acm}^{-2}-3.98 \times 10^{-9}\left[\mathrm{O}_{2}\right]$ (p.p.m. $)$ & 0.9980 & 30 & 90 \\
\hline Microarray sensor & $3,900-39,500$ & $J\left(\mathrm{~A} \mathrm{~cm}^{-2}\right)=-6.44 \times 10^{-6} \mathrm{~A} \mathrm{~cm}^{-2}-9.55 \times 10^{-10}\left[\mathrm{O}_{2}\right]$ (p.p.m.) & 0.9982 & & \\
\hline Macroelectrode sensor ${ }^{\dagger}$ & $3,900-39,500$ & $J\left(\mathrm{Acm}^{-2}\right)=-1.32 \times 10^{-7} \mathrm{~A} \mathrm{~cm}^{-2}-6.68 \times 10^{-11}\left[\mathrm{O}_{2}\right]$ (p.p.m.) & 0.9988 & 1060 & 3,520 \\
\hline
\end{tabular}

A protective container such as a chamber or use of a filter will still be expected for the whole system design to prevent fouling by particulate contaminants.

\section{Discussion}

This work has established facile and reproducible protocols for micropatterning of ILs onto gold and glass surfaces. Several different printing strategies have been developed, namely indirect printing, partial printing, semi-direct printing and direct printing. These demonstrated the capability of the protocol to be customized for different ILs, substrate material in order to target specific applications. Characteristics of the fabricated micropatterns have been investigated by optical, physical and chemical methods, and we concluded that the IL micropatterns are very stable in air and even in some liquids. The excellent mechanical and thermal stabilities are important for commercial applications, including storage and transportation of these microchips.

Micropatterned ILs are demonstrated in this study to be versatile for applications in microfabrication of metals and semiconductors via electrodeposition and photodeposition, and as media for protein immobilization to generate biological microarrays. Benefiting from a number of inherent advantages, including the non-volatility of ILs, their small volume, short diffusion distances to the surface for dissolved molecules and large surface-to-volume ratio, IL microarrays are demonstrated to be promising platforms for membrane-free gas sensors. In principle, analytical procedures or reactions that can be carried 
out in a macro-scale IL system can now be performed at the micro-scale level on a two-dimensional chip. The versatility of the IL microchips paves the way for a wide range of prospective functions that can be explored further for applications such as sensors, microreactor or microfluidic applications.

\section{Methods}

Materials and chemicals. High-purity 1-n-butyl-3-methylimidazolium tetrafluoroborate [BMIM] [BF 4 , 1-n-butyl-3-methylimidazolium bistriflimide [BMIM] $\left[\mathrm{NTf}_{2}\right]$ and 1-n-butyl-3-methylimidazolium hexafluorophosphate $[\mathrm{BMIM}]\left[\mathrm{PF}_{6}\right]$ were purchased from Merck, ethylammonium nitrate (EAN) was purchased from Io-Li-Tec (>97\%). Trichloromethyl silane 99\%, 99\% trichlorohexyl silane, $\geq 99 \%$ triethylamine, $\geq 99 \%$ ethylenediamine, $99 \%$ hexadecanethiol (HDT), 97\% 11-mercaptoundecanoic acid (MUA), 97\% 11-mercapto-1-hexanol (MOH), 99\% 16-PHDA, 98\% 7,7,8,8-tetracyanoquinodimethane (TCNQ), 99.99\% silver tetrafluoroborate $\left(\mathrm{AgBF}_{4}\right)$ and bovine serum albumin-fluorescein isothiocyanate conjugate (BSA-FITC) were purchased from Sigma-Aldrich. Methane sulphonic acid 98\%, and 99.99\% copper nitrate $\left(\mathrm{Cu}\left(\mathrm{NO}_{3}\right)_{2}\right)$ were purchased from Alfa Aesar.

Milli-Q grade reagent water was used throughout the entire experiment for preparation of solutions and rinsing. All the ILs were left open to the air for a week to achieve air equilibration before use.

Synthesis of protic ILs. Protic ILs, triethylammonium methylsulphonate (TAMS), EDAA and EDAF, were synthesized according to the procedure described before ${ }^{36}$. Briefly, the base solution (for example, triethylamine and ethylenediamine) and the acid solution (for example, methylsulphonic acid, acetic acid and formic acid) were purified, dried and handled under inert gas atmosphere. The equivalent value of neat base as well as acid was then added into the reactor simultaneously while stirred vigorously to dissipate the exothermic reaction heat. The addition rate was determined by the reaction volume and was set between 0.2 and $10 \mathrm{ml} \mathrm{min}^{-1}$. The prepared protic IL samples are highly pure, as confirmed by ${ }^{1} \mathrm{H}$ NMR and ${ }^{13} \mathrm{C}$ NMR, and usually contain minimal water content in the range of $\sim 100$ p.p.m.

$\boldsymbol{\mu C P}$ procedures. Polydimethyl siloxane (PDMS) stamps were prepared from Si/SU8 masters and Sylgard 184 PDMS (Dow Corning Corporation) as per the method outlined by Whitesides et al. ${ }^{11}$ Several different $\mu C P$ strategies were tested for fabrication of IL microarray, namely indirect printing, partial printing, semi-direct printing and direct printing.

Indirect printing. In a typical printing experiment, ethanolic solution containing $4 \mathrm{mM}$ of thiols (for example, HDT and MUA) was prepared. A PDMS stamp was inked with one drop of MUA solution for $1 \mathrm{~min}$ and dried gently with nitrogen stream for $30 \mathrm{~s}$ before printing onto a gold substrate. The gap between the patterned MUA SAM was covered by refuelling the slide with HDT. The gold slide was then rinsed twice with ethanol and dried gently with nitrogen stream. A drop of IL (for example, [BMIM] $\left[\mathrm{BF}_{4}\right]$ ) was brought into contact with the patterned area on the gold slides. The excess IL was removed by slow back suction using a micropipette.

Partial printing. The PDMS stamp was inked with ethanolic HDT solution and printed onto a gold substrate without refilling the gaps. The gold slide was rinsed twice with ethanol and dried with gentle nitrogen stream. A drop of IL (for example, $\left.[\mathrm{BMIM}]\left[\mathrm{BF}_{4}\right]\right)$ was brought into contact with the patterned area on the gold slides. The excess IL was removed by slow back suction using a micropipette or by gas blowing with nitrogen.

Semi-direct printing. The PDMS stamp was inked with ethanolic HDT solution and printed onto a gold substrate without refilling the gaps. The gold slide was rinsed twice with ethanol and dried with gentle nitrogen stream. A drop of IL (for example, $\left.[\mathrm{BMIM}]\left[\mathrm{PF}_{6}\right]\right)$ containing $2 \mathrm{mM}$ of MUA was brought into contact with the patterned area on the gold slides. The excess IL was removed by slow back suction using a micropipette.

Direct printing. Microscope glass slides were first cleaned with piranha solution (3:1 concentrated $\mathrm{H}_{2} \mathrm{SO}_{4}$ to $30 \% \mathrm{H}_{2} \mathrm{O}_{2}$ ) for $1 \mathrm{~h}$ (caution: this mixture reacts violently with all organic materials. The solution has to be handled with extreme care to avoid personnel injury and property damage). The slides were rinsed thoroughly with water and dried gently with $\mathrm{N}_{2}$ stream.

The preparation of organophosphanic acid SAM-modified glass surface was according to the protocol described before ${ }^{15}$. Briefly, PHDA monolayers were adsorbed on clean glass surfaces from $5 \mathrm{mM}$ methanol solution for $12 \mathrm{~h}$. The modified substrates were rinsed with methanol and dried with $\mathrm{N}_{2}$, and the substrates were annealed at $150^{\circ} \mathrm{C}$ for $48 \mathrm{~h}$ under $\mathrm{N}_{2}$ to promote stable covalent bonding formation. The annealed surfaces were then sonicated in $0.1 \mathrm{M} \mathrm{K}_{2} \mathrm{CO}_{3}$ for
5 min to remove any possible multilayer and weakly bound molecules. The substrates were thoroughly rinsed with water and dried in $\mathrm{N}_{2}$.

The PDMS stamp was inked with a droplet of IL (for example, EDAA and EDAF). Excessive IL was removed with a lint-free tissue. A thin layer of IL is formed over the PDMS and printed onto a PHDA pre-functionalized glass slide and left for 3 days.

Electrochemical procedures. Cyclic voltammograms were undertaken at $25 \pm 0.2^{\circ} \mathrm{C}$ with a $\mathrm{CH} 760$ Electrochemical Workstation $(\mathrm{CH}$ instrument, Austin, TX, USA) in a three-electrode electrochemical cell. Gold macrodisc electrodes were used as the working electrodes. A platinum wire was used as the counter electrode and a silver or copper wire as the $\mathrm{QRE}$ for $\mathrm{Ag}$ and $\mathrm{Cu}$ deposition, respectively. Before measurements, the working electrodes were polished with $0.05 \mu \mathrm{m}$ alumina slurry ( $\mathrm{CH}$ instrument) on a clean polishing cloth (Buehler, Lake Bluff, IL, USA), sequentially rinsed with distilled water and acetone, and finally dried with lint-free tissue.

The on-chip electrodeposition was carried out using the open microelectrochemical cell configuration illustrated in Supplementary Fig. 6. A SAMmodified gold chip fabricated by chemical vapour deposition was used as the working electrode. A Ag wire QRE and a Pt wire counter electrode were bought carefully in contact with the IL reservoirs at the edge of the IL pattern, without touching the working electrode. Controlled potential electrodeposition of $\mathrm{Ag}, \mathrm{Cu}$ and AgTCNQ were performed using the gold electrode according to the procedure described before ${ }^{23-25}$.

Photochemical procedures. The irradiation procedure was modified from the procedure described before ${ }^{37}$. [BMIM] $\left[\mathrm{BF}_{4}\right]$ solution containing $3.33 \mathrm{mM}$ of $\mathrm{AgBF}_{4}, 3.33 \mathrm{mM}$ of TCNQ and $10 \% \mathrm{v} / \mathrm{v}$ of benzyl alcohol was prepared by sonication for $30 \mathrm{~min}$. The IL solution was patterned onto gold surfaces and irradiated at $\lambda=425 \mathrm{~nm}$ for $3 \mathrm{~h}$ using a Solar Simulator 50-500 W arc lamp 69907 (Newport, Stratford) coupled with a filter.

Fabrication of protein microarray procedures. IL solution containing $10 \mu \mathrm{M}$ of Cyt $c$-OG488 or $0.75 \mu \mathrm{M}$ of BSA-FITC and $10 \% \mathrm{v} / \mathrm{v}$ of phosphate-buffered saline buffer dissolution was prepared by vortexing for $5 \mathrm{~min}$. The IL/protein solution was then patterned onto the gold slide via the indirect printing technique.

Gas sensor experiments. A gas sensor system was set up and is illustrated in Supplementary Fig. 16. Ultra-high purity $\mathrm{N}_{2}$ and $\mathrm{O}_{2}$ were purchased from Air Liquide Australia (New South Wales, Australia). The flow rates and concentration of the $\mathrm{O}_{2}$ were controlled and calculated by using four flowmeters (Cole-Palmer, New South Wales, Australia). The mixed gas was allowed to pass through a gas-mixing segment to create gas turbulence to ensure adequate mixing before entering the detection chamber. The system allows preparation of $\mathrm{O}_{2}$ gas samples in the range from min. 130 p.p.m. to max. $100 \%$.

Amperometric gas sensing experiments were carried out using the similar electrochemical setup to metal deposition illustrated in Supplementary Fig. 6 . A hydrophobic aprotic IL, 1-n-butyl-3-methylimidazolium bistriflimide $[\mathrm{BMIM}]\left[\mathrm{NTf}_{2}\right]$ was patterned onto the gold macrodisc working electrode via partial printing to form 30- $\mu \mathrm{m}$-wide IL lines with $60 \mu \mathrm{m}$ spacing. The effective surface area of the bare gold macrodisc electrode was measured to be $0.035 \mathrm{~cm}^{2}$ using the cyclic voltammetry method ${ }^{34}$. After printing, the IL covered approximately one-third of the total area of the bare gold electrode, and the effective area of electrode in contact with IL microarray was calculated to be $0.012 \mathrm{~cm}^{2}$.

For comparison purpose, a conventional type, macroelectrode-IL gas sensor was fabricated using a gold macroelectrode of same size $\left(0.035 \mathrm{~cm}^{2}\right)$, according to the method described in the literature ${ }^{38}$. Briefly, a thin layer of IL was applied to cover the gold macrodisc electrode. The thickness of the IL layer is calculated to be $100 \pm 10 \mu \mathrm{m}$ from volume of the IL applied and the inner diameter of the electrochemical cell (Supplementary Fig. 16). It should be noted that the thickness of the IL layer plays a significant role in the current responses ${ }^{39}$, and $100 \mu \mathrm{m}$ thickness used in study is derived from the minimal volume of ILs that ensures a full coverage of the macroelectrode. It is also worth noting that the actual thickness of the IL layer above the electrode could be smaller than $100 \mu \mathrm{m}$ owing to the formation of a meniscus at the edge of the cell walls.

Before the experiments, the gas chamber was purged with nitrogen for $30 \mathrm{~min}$ to remove any traces of $\mathrm{O}_{2}$ impurity that may presence in the systems. A constant potential of $-1.2 \mathrm{~V}$ was applied to ensure that $\mathrm{O}_{2}$ reduction is mass transport limited, hence all the analytes that reach the electrode will be reduced simultaneously. Under these conditions, the sensitivity of the sensor is governed mainly by the gas flow rate through the tubing, and $\mathrm{O}_{2}$ diffusion rate from the gas phase inside the flask to the surface of the electrode ${ }^{40}$. Once a stable baseline was achieved, the $\mathrm{O}_{2}$ sample was introduced into the systems. The $\mathrm{O}_{2}$ reduction current was recorded as a function of time, and the response times were taken from the time the sensor started to respond after the gas was applied. The characteristic $T_{50}$ and $T_{90}$ values for each sensor type are measured from the time when the sensor response current reaches $50 \%$ and $90 \%$ of its steady-state value, respectively. It should be noted that response time of a gas sensor is strongly dependent on the diffusion properties of each sensor such as tubing length, flow rates, diffusion 
barriers and so on, and direct comparison between literatures are usually difficult ${ }^{40}$. Our study applied the same sensor configurations and conditions for different sensing electrode designs, which allows for sensible comparisons.

Physical characterizations. All reflectance micrographs were recorded using a Nikon eclipse TS100-F inverted microscope (Nikon, Japan). All phase contrast micrographs were recorded using a Nikon eclipse LV150L microscope (Nikon). Scanning confocal microscopy measurements were performed using Zeiss LSM 780 (Oberkochen, Germany).

IR spectroscopy measurements were performed by Perkinelmer Spotlight 400 FTIR microscope. The recorded data were connected relative to the gold surface on reflectance mode with $\times 64 \mathrm{scan}, 16 \mathrm{~cm}^{-1}$ resolution for the single-point detection. SEM measurements were performed by FEI Nova SEM 230 with a Bruker Energy Dispersive X-Ray (EDX) system operated on $10 \mathrm{kV}$ accelerating voltage.

The fluorescence images were taken by using a Leica DM IL LED inverted confocal microscope (Leica Microsystems, Buffalo Grove, IL, USA) or a GFP fluorescence slider (excitation filter: BP470/40, dichroic mirror: 500 and emission filter: 525/50), a mercury short-arc reflector lamp and a ProgRes CFscan camera (Jenoptik Laser, Optik, Systeme GmbH, Germany).

\section{References}

1. Hogan, J. Lab on a chip: a little goes a long way. Nature 442, 351-352 (2006).

2. Dubois, P. et al. Ionic liquid droplet as e-microreactor. Anal. Chem. 78, 4909-4917 (2006).

3. Ding, H. J. et al. Accurate dispensing of volatile reagents on demand for chemical reactions in EWOD chips. Lab Chip 12, 3331-3340 (2012).

4. Dubois, P., Marchand, G., Gmouh, S. \& Vaultier, M. Reaction rates as a function of scale within ionic liquids: microscale in droplet microreactors versus macroscale reactions in the case of the grieco three-component condensation reaction. Chem. Eur. J. 13, 5642-5648 (2007).

5. Fair, R. B. Digital microfluidics: is a true lab-on-a-chip possible? Microfluid. Nanofluid. 3, 245-281 (2007)

6. Freemantle, M. An Introduction to Ionic Liquids (Royal Society of Chemistry, 2009).

7. Hozumi, A., Bien, P. \& McCarthy, T. J. Ionic liquids: nondestructive, nonvolatile imaging fluids for submicrometer-scale monolayer patterns. J. Am. Chem. Soc. 132, 5602-5603 (2010).

8. Huang, X.-J., Aldous, L., O’Mahony, A. M., del Campo, F. J. \& Compton, R. G. Toward membrane-free amperometric gas sensors: a microelectrode array approach. Anal. Chem. 82, 5238-5245 (2010).

9. Marchand, G. et al. Organic synthesis in soft wall-free microreactors: real-time monitoring of fluorogenic reactions. Anal. Chem. 80, 6051-6055 (2008).

10. Jeong, J. et al. Suppression of metal-insulator transition in $\mathrm{VO}_{2}$ by electric field-induced oxygen vacancy formation. Science 339, 1402-1405 (2013).

11. Qin, D., Xia, Y. \& Whitesides, G. M. Soft lithography for micro- and nanoscale patterning. Nat. Protoc. 5, 491-502 (2010).

12. Awada, H., Castelein, G. \& Brogly, M. Quantitative determination of surface energy using atomic force microscopy: the case of hydrophobic/hydrophobic contact and hydrophilic/hydrophilic contact. Surf. Interface Anal. 37, 755-764 (2005).

13. Li, J., Shen, Y., Zhang, Y. \& Liu, Y. Room-temperature ionic liquids as media to enhance the electrochemical stability of self-assembled monolayers of alkanethiols on gold electrodes. Chem. Commun. 3, 360-362 (2005).

14. Lu, X., Burrell, G., Separovic, F. \& Zhao, C. Electrochemistry of room temperature protic ionic liquids: a critical assessment for use as electrolytes in electrochemical applications. J. Phys. Chem. B 116, 9160-9170 (2012).

15. Chockalingam, M., Darwish, N., Le Saux, G. \& Gooding, J. J. Importance of the indium tin oxide substrate on the quality of self-assembled monolayers formed from organophosphonic acids. Langmuir 27, 2545-2552 (2011).

16. Goel, A. Surface Chemistry (Discovery Publishing House, 2006).

17. Kim, K.-S., Demberelnyamba, D. \& Lee, H. Size-selective synthesis of gold and platinum nanoparticles using novel thiol-functionalized ionic liquids. Langmuir 20, 556-560 (2004)

18. Chen, G., Paronyan, T. M., Pigos, E. M. \& Harutyunyan, A. R. Enhanced gas sensing in pristine carbon nanotubes under continuous ultraviolet light illumination. Sci. Rep. 2, 343 (2012).

19. Lynnette, A. B., Dan, H., Eric, J. B. \& Joan, F. B. Green processing using ionic liquids and $\mathrm{CO}_{2}$. Nature 399, 28-29 (1999).

20. Jeon, Y. et al. Structures of ionic liquids with different anions studied by infrared vibration spectroscopy. J. Phys. Chem. B 112, 4735-4740 (2008).

21. Frédéric, R., Fn, David., Hye Jin, L. \& Hubert, H. G. Electrochemistry at liquid/liquid interfaces: methodology and potential applications. Electrochim. Acta 45, 2647-2662 (2000).

22. Endres, F., MacFarlane, D. R. \& Abbott, A. Electrodeposition from Ionic Liquid (Wiley-VCH, 2008)
23. Zhao, C., MacFarlane, D. R. \& Bond, A. M. Modified thermodynamics in ionic liquids for controlled electrocrystallization of nanocubes, nanowires, and crystalline thin films of silver - tetracyanoquinodimethane. J. Am. Chem. Soc. 131, 16195-16205 (2009).

24. Gunawan, C. A., Suryanto, B. H. R. \& Zhao, C. Electrochemical study of copper in room temperature protic ionic liquids ethylammonium nitrate and triethylammonium methylsulfonate. J. Electrochem. Soc. 159, D611-D615 (2012).

25. Suryanto, B. H. R., Gunawan, C. A., Lu, X. \& Zhao, C. Tuning the electrodeposition parameters of silver to yield micro/nano structures from room temperature protic ionic liquids. Electrochim. Acta 81, 98-105 (2012)

26. Zhao, C. \& Bond, A. M. Photoinduced oxidation of water to oxygen in the ionic liquid BMIMBF4 as the counter reaction in the fabrication of exceptionally long semiconducting silver-tetracyanoquinodimethane nanowires. J. Am. Chem. Soc. 131, 4279-4287 (2009).

27. Castner, E., Margulis, C., Maroncelli, M. \& Wishart, J. Ionic liquids: structure and photochemical reactions. Annu. Rev. Phys. Chem. 62, 85-105 (2011).

28. Plechkova, N. V., Rogers, R. D. \& Seddon, K. R. Ionic Liquids: From Knowledge to Application (American Chemical Society, 2010).

29. Armand, M., Endres, F., MacFarlane, D. R., Ohno, H. \& Scrosati, B. Ionic-liquid materials for the electrochemical challenges of the future. Nat. Mater. 8, 621-629 (2009).

30. Kyoko, F., Douglas, R. M. \& Maria, F. Protein solubilising and stabilising ionic liquids. Chem. Commun. 38, 4804-4806 (2005).

31. Moniruzzaman, M., Kamiya, N. \& Goto, M. Activation and stabilization of enzymes in ionic liquids. Org. Biomol. Chem. 8, 2887-2899 (2010).

32. Buzzeo, M., Hardacre, C. \& Compton, R. Use of room temperature ionic liquids in gas sensor design. Anal. Chem. 76, 4583-4588 (2004).

33. Huang, X.-J., Rogers, E., Hardacre, C. \& Compton, R. The reduction of oxygen in various room temperature ionic liquids in the temperature range $293-318 \mathrm{~K}$ : exploring the applicability of the Stokes-Einstein relationship in room temperature ionic liquids. J. Phys. Chem. B 113, 8953-8959 (2009).

34. Khan, A., Lu, X., Aldous, L. \& Zhao, C. Oxygen reduction reaction in room temperature protic ionic liquids. J. Phys. Chem. C 117, 18334-18342 (2013).

35. Shrivastava, A. \& Gupta, V. Methods for the determination of limit of detection and limit of quantitation of the analytical methods. Chron. Young Scientists 2 , 21-25 (2011).

36. Burrell, G. L., Burgar, I. M., Separovic, F. \& Dunlop, N. F. Preparation of protic ionic liquids with minimal water content and 15N NMR study of proton transfer. Phys. Chem. Chem. Phys. 12, 1571-1577 (2010).

37. O'Mullane, A. P., Fay, N., Nafady, A. \& Bond, A. M. Preparation of Metal-TCNQ charge-transfer complexes on conducting and insulating surfaces by photocrystallization. J. Am. Chem. Soc. 129, 2066-2073 (2007).

38. Ji, X. B., Silvester, D. S., Aldous, L., Hardacre, C. \& Compton, R. G. Mechanistic studies of the electro-oxidation pathway of ammonia in several roomtemperature ionic liquids. J. Phys. Chem. C 111, 9562-9572 (2007).

39. Zevenbergen, M., Wouters, D., Dam, V-AT., Brongersma, S. \& Crego-Calama, $\mathrm{M}$. Electrochemical sensing of ethylene employing a thin ionic-liquid layer. Anal. Chem. 83, 6300-6307 (2011).

40. Warburton, P. et al. Amperometric gas sensor response times. Anal. Chem. 70, 998-1006 (1998).

\section{Acknowledgements}

We are grateful to colleagues at the School of Chemistry, The University of New South Wales, Professor Justin Gooding and A/Professor Pall Thordarson, for sharing their Si/SU8 masters and Cyt $c$-OG488, respectively, Professor Brynn Hibbert for helpful discussions and Mr Alex Mason for the help in fluorescence microscopy, and UNSW Mark Wainwright Analytical Center for providing access to their imaging facilities. The study was financed by the Australian Research Council (DP120101604).

\section{Author contributions}

C.Z. directed the project. C.Z. and C.A.G. planned the experiments, analysed the data and co-wrote the paper. C.A.G and M.G. performed the experiments.

\section{Additional information}

Supplementary Information accompanies this paper at http://www.nature.com/ naturecommunications

Competing financial interests: The authors declare no competing financial interests

Reprints and permission information is available online at http://npg.nature.com/ reprintsandpermissions/

How to cite this article: Gunawan, C. A. et al. Robust and versatile ionic liquid microarrays achieved by microcontact printing. Nat. Commun. 5:3744 doi: 10.1038/ncomms4744 (2014). 